\title{
Graphs Associated with Codes of Covering Radius 1 and Minimum Distance 2
}

\author{
Joanne L. Hall \\ School of Mathematical and Geospatial Sciences \\ Royal Melbourne Institute of Technology \\ joanne.hall@rmit.edu.au \\ Department of Mathematics \\ Australian National University
}

Submitted: Dec 10, 2007; Accepted: Apr 23, 2008; Published: May 5, 2008

Mathematics Subject Classifications: 94B65, 05C15, 05B15

The search for codes of covering radius 1 led Östergård, Quistorff and Wassermann to the OQW method of associating a unique graph to each code [9]. We present results on the structure and existence of OQW-associated graphs. These are used to find an upper bound on the size of a ball of radius 1 around a code of length 3 and minimum distance 2. OQW-associated graphs and non-extendable partial Latin squares are used to catalogue codes of length 3 over 4 symbols with covering radius 1 and minimum distance 2 .

\section{Introduction}

The search for the minimum or maximum cardinality of an object with a set of properties is one of the classical problems in combinatorics. An $(n, V, d)_{q} R$ code, $\mathcal{C}$, is a code of length $n$ over $q$ symbols with $V$ codewords, a minimum Hamming distance of $d$ and covering radius of $R$. $(n, V, d)_{q}$ or $(n, V)_{q} R$ are used if the covering radius or minimum distance are not specified.

Let $K_{q}(n, R)$ be the minimum $V$ such that an $(n, V)_{q} R$ code exists, and $A_{q}(n, d)$ be the maximum $V$ such that an $(n, V, d)_{q}$ code exists. The problems of finding $K_{q}(n, R)$ and $A_{q}(n, d)$ are well studied. The Delsarte bound [4] and the Hamming bound [7] are some well know early results. For more recent studies see $[2,8]$.

The study of codes with both specified covering radius and minimum distance is more recent and has mostly been studied using covering radius 1 and minimum distance 2 $[10,9,1,5] .(n, V, 2)_{q} 1$ codes are also of interest as they are equivalent to non-extendable partial Latin hypercubes [5]. 


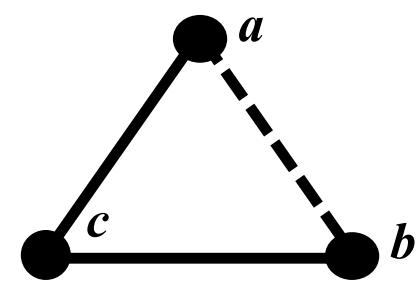

Figure 1: A graph which has no OQW-associated code.

This article provides a summary and extension of the author's thesis [6].

Let $K_{q}(n, R, d)\left(A_{q}(n, R, d)\right)$ be the minimum (maximum) $V$ such that an $(n, V, d)_{q} R$ code exists. $K_{q}(n, R) \leq K_{q}(n, R, d)$ and $A_{q}(n, d) \geq A_{q}(n, R, d)$; it is an open problem to determine for which parameters these inequalities are strict.

For $n \leq 3, K_{q}(n, 1,2)=K_{q}(n, 1)[10]$. Using the sphere covering bound $K_{2}(4,1)=$ $K_{2}(4,1,2)=4$ and $K_{3}(4,1)=K_{3}(4,1,2)=9$ [9]. $K_{4}(4,1)=24$ [12]. [9] shows $K_{4}(4,1,2) \geq 25$ by associating a unique graph to each code.

Definition 1. [9] A graph $\mathcal{G}$ and a code $\mathcal{C}$ are OQW-associated if, and only if, each vertex of $\mathcal{G}$ represents a codeword of $\mathcal{C}$, and an edge of colour $\alpha$ connects two vertices of $\mathcal{G}$ if, and only if, the two codewords of $\mathcal{C}$ represented have the same symbol in the $\alpha^{\text {th }}$ position.

In section 2 we give general properties of OQW-associated graphs. In section 3 OQWassociated graphs are used to find an upper bound on the size of a ball of radius 1 around $(3, V, 2)_{q}$ codes. In section 4 we find properties of codes which meet the bound. In section 5 we almost completely catalogue $(3, V, 2){ }_{4} 1$ codes.

\section{OQW-Association Method}

Let $\mathcal{G}_{\alpha}$ be the subgraph of $\mathcal{G}$ containing all the vertices of $\mathcal{G}$ but only the edges of colour $\alpha$. We are only interested in the structure of colours within the graph; permuting colours will create an equivalent graph.

Every code (up to equivalence) has a unique (up to equivalence) OQW-associated graph. (We ignore codes where every codeword has the same symbol in position $\alpha$.) Not every graph has an OQW-associated code, e.g. figure 1.

Theorem 2. [6, Thm 2.6] A graph $\mathcal{G}$ is $O Q W$-associated with $\mathcal{C}$, an $(n, V, d)_{q}$ code if, and only if, it satisfies the following five properties.

P1 $\mathcal{G}$ has $V$ vertices.

P2 $n$ is greater than or equal to the number of colours of edges of $\mathcal{G}$.

P3 There are no more than $n-d$ edges between any two vertices.

$\mathbf{P} 4$ Any connected subgraph of any $\mathcal{G}_{\alpha}$ is complete, and $q^{n-d}$ is greater than or equal to the number of vertices in the largest single coloured connected subgraph. 
P5 $q$ is greater than or equal to the number of connected components of any $\mathcal{G}_{\alpha}, \alpha \in$ $\{1, \ldots, n\}$.

Before proving this result we need a few preliminary lemmas.

Lemma 3. [6, Lem 2.8] Let $\mathcal{G}$ be a graph $O Q W$-associated with an $(n, V, d)_{q}$ code, $\mathcal{C}$. Suppose we add a vertex $v$ and some edges incident with $v$ (of any colour) to form $\mathcal{G}^{\prime}$. If for each $\alpha$, any connected subgraph of $\mathcal{G}_{\alpha}^{\prime}$ is a complete graph, then $\mathcal{G}^{\prime}$ will have an $O Q W$-associated code $\mathcal{C}^{\prime}$.

Proof. Adding vertex $v$ to $\mathcal{G}$ adds a word to $\mathcal{C}$ to form $\mathcal{C}^{\prime}$. As every connected component of each $\mathcal{G}_{\alpha}^{\prime}$ is a complete graph, the word is uniquely determined by the edges incident with $v . \mathcal{C}^{\prime}$ is an $\left(n^{\prime}, V+1, d^{\prime}\right)_{q^{\prime}}$ code with $q^{\prime} \geq q, d^{\prime} \leq d$ and $n^{\prime} \geq n$.

Lemma 4. [6, Lem 2.7] Let $\mathcal{G}$ be a graph on $V$ vertices that satisfies P1-P5. Suppose we remove a vertex $v$ and any edges incident with $v$ to form $\mathcal{G}^{\prime}$, then $\mathcal{G}^{\prime}$ will also satisfy P1-P5.

Proof. P1, P2 are obvious. P3, P4, P5: $\mathcal{G}^{\prime}$ is a subgraph of $\mathcal{G}$, thus the number of colours of $\mathcal{G}^{\prime}$ and the size and number of connected components of $\mathcal{G}_{\alpha}^{\prime}$ cannot be any larger than that of $\mathcal{G}$ or $\mathcal{G}_{\alpha}$.

Now we are ready for the proof of Theorem 2 .

P1. The vertices of $\mathcal{G}$ and words of $\mathcal{C}$ are in a bijective correspondence.

P2. Each colour of edges represents a specific position in the code. Therefore the length of the code can be no less than the number of colours.

P3. Any two codewords can have the same symbol in at most $n-d$ positions. Thus there can be at most $n-d$ edges between any two vertices.

P4. The relationship expressed by edges is transitive; any vertices connected by a single coloured path, must be connected by an edge of that colour. Any two codewords must differ in at least $d$ places, so there can be at most $q^{n-d}$ codewords with the same symbol in position $\alpha$.

P5. Each of the connected components of $\mathcal{G}_{\alpha}$ are complete graphs which represent the set of codewords with the same symbol in the $\alpha^{\text {th }}$ position. Each component must have a different symbol.

We have shown that P1-P5 are necessary. We proceed by induction on $V$ to show they are sufficient.

Begin with $V=1: K_{1}$, the complete graph on 1 vertex, satisfies P1-P5 and has an OQW-associated code. Suppose every graph $\mathcal{G}$ which satisfies P1-P5 with $V \leq m$ vertices has an OQW-associated code.

Take a graph $\mathcal{G}$ with $m+1$ vertices that satisfies P1-P5. Let $\mathcal{G}^{\prime}$ be a graph obtained by removing a vertex and its incident edges from $\mathcal{G}$. From Lemma 4 we know that $\mathcal{G}^{\prime}$ will also satisfy P1-P5. By the inductive hypothesis $\mathcal{G}^{\prime}$ has an OQW-associated code.

Using Lemma 3 we can add back the vertex and edges to form $\mathcal{G}$, which must have an OQW-associated code. Thus by induction every graph which satisfies P1-P5 has an OQW-associated code.

We name the class of graphs which can be OQW-associated with a code OQW-graphs. 


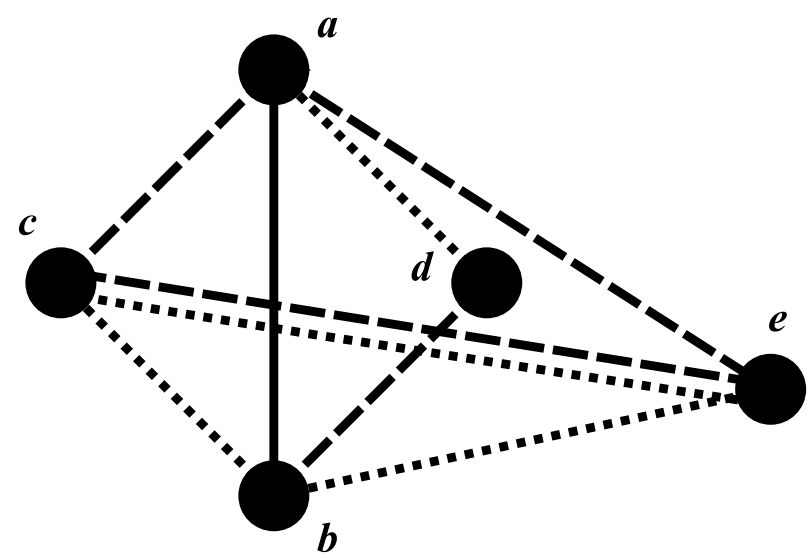

Figure 2: Edge $(a, b)$ in three multicoloured triangles requires a double edge.

\section{Bound on $\left|B_{1}(\mathcal{C})\right|$}

We now know the defining characteristics of the OQW-graphs, and can use them in the search for $K_{q}(n, R, d)$ and $A_{q}(n, R, d)$. $B_{R}(\mathcal{C})$, the ball of radius $R$ around a code is the union of balls of radius $R$ around the codewords. We look for a bound on $\left|B_{R}(\mathcal{C})\right|$.

A multicoloured triangle has more than one colour of edges. Because the relationship expressed by edges is transitive, all triangles in an OQW-graph are single coloured or have three different coloured edges. The following result is a simple application of inclusionexclusion.

Theorem 5. [9, Theorem 3] Let $\mathcal{C}$ be a $(3, V, 2)_{q}$ code with $\mathcal{G}$, its $O Q W$-associated graph having $V$ vertices and $E$ edges. Let $T$ be the number of multicoloured triangles in $\mathcal{G}$, then

$$
\left|B_{1}(\mathcal{C})\right|=(1+3(q-1)) V-2 E+T
$$

Next we find a bound on the number of multicoloured triangles and edges in a graph, then a bound on $\left|B_{1}(\mathcal{C})\right|$. This bound is sharp.

Lemma 6. [6, Lem 3.4, with Brendan McKay] Let $\mathcal{G}$ be a graph with E edges that is $O Q W$-associated with a $(3, V, 2)_{q}$ code. Then $T \leq \frac{2}{3} E$.

Proof. Let an edge $(a, b)$ be in two multicoloured triangles. Then there are two vertices $c, d$ each forming the third vertex of a triangle. To this we add another vertex $e$ to form a multicoloured triangle with edge $(a, b)$ as in figure 2 .

Edges represent a transitive relationship, so a double edge is required to form single coloured triangles with vertex $c$. This contradicts P3. Thus each edge can be in at most two multicoloured triangles.

Note this only holds for $n=3$. For a more general result see [6, §4]. 

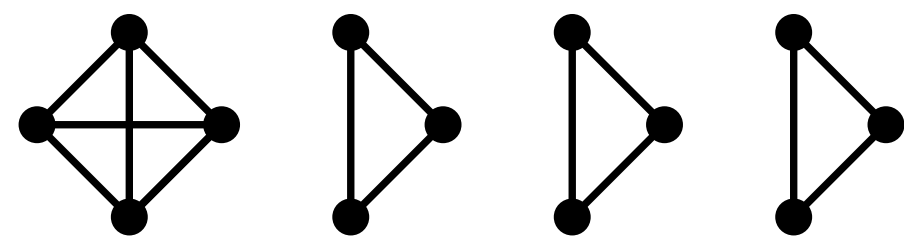

Figure 3: Minimum number of edges of $\mathcal{G}_{a}$ for a $(3,13,2)_{4}$ code.

Lemma 7. [6, Cor 2.9] $\mathcal{G}$ is a graph $O Q W$-associated with an $(n, V, n-1)_{q}$ code. Let $V=z q+w$ where $z \geq 0$ and $0 \leq w<q$. Then $\mathcal{G}$ has at least

$$
\frac{q}{2}(z-1) z+w z
$$

edges of each colour.

Proof. The minimum number of edges occurs when $\mathcal{G}_{\alpha}$ consists of complete graphs of lowest degree. There are $q$ connected components of $\mathcal{G}_{\alpha}$. Each component has at least $z$ vertices, with $w$ of the components having $z+1$ vertices. E.g. figure 3. There are $q K_{z} \mathrm{~s}$ each having $\frac{1}{2}(z-1) z$ edges. There are $w$ of the components with an extra vertex, and hence an extra $z$ edges.

Theorem 8. [6, Thm 3.7] Let $\mathcal{C}$ be a $(3, V, 2)_{q}$ code. Let $V=z q+w$ with $z \geq 0$ and $0 \leq w<q$ then

$$
\left|B_{1}(\mathcal{C})\right| \leq(1+3(q-1)) V-\frac{4}{3}\left(\frac{3 q}{2}(z-1) z+z w\right) .
$$

Proof. From Lemma 6 and Theorem 5 we find that

$$
\left|B_{1}(\mathcal{C})\right| \leq(1+3(q-1)) V-\frac{4}{3} E .
$$

We then use Lemma 7, to obtain inequality (3).

For $V \geq \frac{1}{2} q^{2}$ inequality 3 becomes larger than $q^{3}$ which is the size of the Hamming space. Thus Theorem 8 is only relevant for $V \leq \frac{1}{2} q^{2}$.

\section{Codes which Meet the Upper Bound}

Inequality (3) gives us a good bound on $\left|B_{1}(\mathcal{C})\right|$ for $(3, V, 2)_{q}$ codes where $V \leq \frac{1}{2} q^{2}$ (For $V \geq \frac{1}{2} q^{2}$ inequality (3) becomes larger than the size of the Hamming space.). This bound can only be achieved if every edge in $\mathcal{G}$ is in two multicoloured triangles and there is a minimum number of edges. We now look at other properties the graph must have in order to meet the bound of inequality (3).

Theorem 9. If $\mathcal{G}$ is a graph $O Q W$-associated with a $(3, V, 2)_{q}$ code, such that every edge is in two multicoloured triangles then the following hold: 


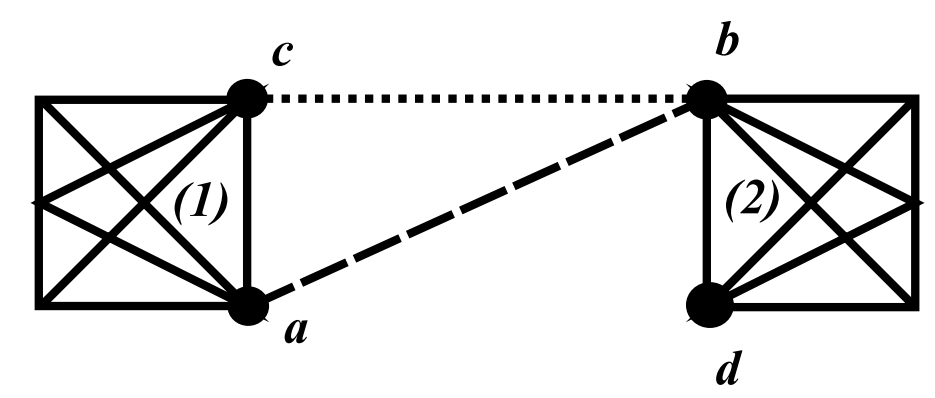

Figure 4: Edge $(a, b)$ links different $(j+1)$-cliques of colour $\beta$.

M1 [6, Thm 3.9-1] Each connected component of $\mathcal{G}$ is 3j-regular, for some integer $j$.

M2 [6, Thm 3.9-2] The number of vertices of each connected component of $\mathcal{G}$ must be exactly $(j+1)^{2}$.

M3 [6, Thm 3.9-3] Any two adjacent vertices of $\mathcal{G}$ are mutually adjacent to $j+1$ other vertices.

M4 Any two non-adjacent vertices of a connected component of $\mathcal{G}$ are mutually adjacent to six other vertices.

Proof. M1. Let edge $(a, b)$ of colour $\alpha$ be in two multicoloured triangles. Vertices $a$ and $b$ must each be incident with an edge of each of colours $\beta$ and $\gamma$. Let another edge $(a, e)$ of colour $\beta$ be added. If no further edges of colours $\alpha$ and $\gamma$ are incident with $a$, then in order for edge $(a, e)$ to be in two multicoloured triangles, edge $(a, b)$ must be included in one of them. This however requires a double edge, (figure 2). Thus another edge of colour $\alpha$ incident with vertex $a$ must be added. Repeating this process for each edge we conclude that each vertex must be incident with the same number of edges of each colour.

If a vertex $a$ has $x$ edges of colour $\alpha$ incident with it, then by $\mathrm{P} 4$ every vertex adjacent to $a$ by an edge of colour $\alpha$ will also have $x$ edges of colour $\alpha$ incident with it. Thus all vertices in any connected component must be incident with an equal number of edges of each colour.

M2. Each $\mathcal{G}_{\alpha}$ is a collection of disjoint $K_{(j+1)} s$. Hence the number of vertices must be a multiple of $(j+1)$.

Choose an edge $(a, b)$ of colour $\alpha$ that is in a multicoloured triangle. This edge connects two vertices which are in different $(j+1)$-cliques of colour $\beta\left(K_{(j+1)}(1), K_{(j+1)}(2)\right)$, and is adjacent to an edge $(b, c)$ of colour $\gamma$ which also connects $K_{(j+1)}(1)$ and $K_{(j+1)}(2)$. E.g. figure 4.

An edge of colour $\gamma$ must include either $a$ or $b$ to be in a multicoloured triangle with $(a, b)$. Without loss of generality we choose edge $(a, d)$. All edges of colour $\beta$ adjacent to $(a, b)$ are in $K_{(j+1)}(1)$ or $K_{(j+1)}(2)$, and all edges that are incident with $a$ are in $K_{(j+1)}(1)$. Thus to complete the multicoloured triangle without creating a double edge, $d$ must be in $K_{(j+1)}(2)$. 
Thus every $(j+1)$-clique must be adjacent to every other $(j+1)$-clique; there must be $j+1(j+1)$-cliques of each colour.

M3. If two vertices $a$ and $b$ are adjacent, and we count the number of mutually adjacent vertices, then we are counting the number of triangles which involve $a$ and $b$. Every edge is in two multicoloured triangles, thus has two mutually adjacent vertices through multicoloured triangles. Any two vertices are in a single coloured $(j+1)$-clique and therefore have $j-1$ mutually adjacent vertices through single coloured triangles.

M4. Assume edge $(a, b)$ of colour $\alpha$ is in two multicoloured triangles involving vertices $c$ and $d$. If vertices $c$ and $d$ are non-adjacent, they are mutually adjacent to vertex $a$ through a path of colours $\beta \gamma$ and vertex $b$ through a path colours of $\gamma \beta$. There are six connecting path colours: $\alpha \beta, \alpha \gamma, \beta \alpha, \beta \gamma, \gamma \alpha, \gamma \beta$. Edge $(a, c)$ is in two multicoloured triangles involving vertices $b$ and $f$. $a$ and $c$ are mutually adjacent to $f$ through a path of colour $\alpha \gamma$. Continuing in the same manner all of the path colours appear. There can be no paths which share the same colours, otherwise a double edge would be required as in the paths from vertices $a$ and $b$ in figure 2 .

These four individual properties combine to the following result.

Theorem 10. If $\mathcal{G}$ is a graph $O Q W$-associated with a $(3, V, 2)_{q}$ code, such that every edge is in two multicoloured triangles then each connected component of $\mathcal{G}$ is a $\left((j+1)^{2}, 3 j, j+\right.$ $1,6)$ strongly regular graph.

Strongly regular graphs have been completely catalogued for many small sizes [11]. The graphs must be checked for an appropriate colouring.

\section{A catalogue of $(3, V, 2)_{4} 1$ codes}

We use OQW-graphs and non-extendable partial Latin squares to almost completely catalogue $(3, V, 2){ }_{4} 1$ codes.

Definition 11. A partial Latin square is an $n \times n$ array in which each cell contains an element of the symbol set $\{0,1, \ldots, n-1\}$ or is empty, so that each symbol occurs at most once in each row and column. A partial Latin square is non-extendable if for each empty cell, every element of the symbol set appears in the row or column of that cell.

We mark empty cells with an X. If there are no empty cells then the array may be called a Latin square. If a partial Latin square has an empty row or column, then it is extendable.

A $(3, V, 2)_{4} 1$ code is equivalent to a non-extendable partial Latin square of side length 4 with $16-V$ blank cells [5]. E.g. figure 5 .

Let $\mathcal{L}$ be the partial Latin square equivalent to the code $\mathcal{C}$. A $2 \times 2$ Latin subsquare in $\mathcal{L}$ is equivalent to a multicoloured 4 -clique in $\mathcal{G}$ the OQW-associated graph. Main class equivalence [3] of Latin squares corresponds to equivalence in codes: permutation of the rows, the columns and the symbols in $\mathcal{L}$ corresponds to permuting the symbols in each of the 3 positions in $\mathcal{C}$; permutation on the set $\{$ row, column, symbol $\}$ corresponds 


\begin{tabular}{|c|c|c|c|}
\hline 0 & 1 & 2 & $\mathrm{X}$ \\
\hline 1 & 0 & $\mathrm{X}$ & 3 \\
\hline 2 & 3 & 1 & 0 \\
\hline 3 & $\mathrm{X}$ & 0 & 2 \\
\hline
\end{tabular}

Figure 5: The partial Latin square equivalent to the code $\{000,011,022,101,110,133,202,213,221,230,303,320,332\}$.
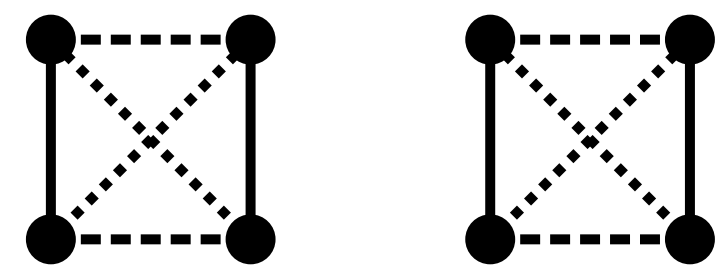

Figure 6: Graph associated with the $(3,8,2)_{4} 1$ code.

to permuting the positions in $\mathcal{C}$, or equivalently the colours of $\mathcal{G}$. We use R,C,S to label row, column and symbol permutations.

$K_{4}(3,1,2)=8[10]$ and using the Singleton bound $A_{4}(3,1,2)=16$. We completely catalogue $(3, V, 2){ }_{4} 1$ codes where $V=8,9,10,11,13,14,15,16$ and provide some examples for $V=12$.

Theorem 12. [6, Thm 3.14] There is a unique $(3,8,2)_{4} 1$ code.

Proof. Figure 6 gives an example of an OQW-associated graph that meets $K_{4}(3,1,2)=8$. Using Theorem 9 this is unique.

Theorem 13. [6, Thm 3.16] No $(3,9,2){ }_{4} 1$ code exists.

Proof. From Lemma 7 we know that $\mathcal{G}$, the OQW-associated graph, must have one triangle and 3 isolated edges of each colour. $\mathcal{L}$, the equivalent Latin square, must have at least one empty cell in each row and column, with 3 of the rows and columns having 2 empty cells. There are three possible structures of $\mathcal{L}$ depending on if we allow a vertex of order 6 in $\mathcal{G}$ (figure 7 ).

We can begin by filling in row 3 and column 1 . For $\mathcal{L} A$ and $\mathcal{L} B$ : to ensure cell $(2,1)$ cannot be filled, row 2 may be filled uniquely. From this row 2 and column 2 cannot possibly contain all symbols unless one of the cells marked with an $\mathrm{X}$ is filled.
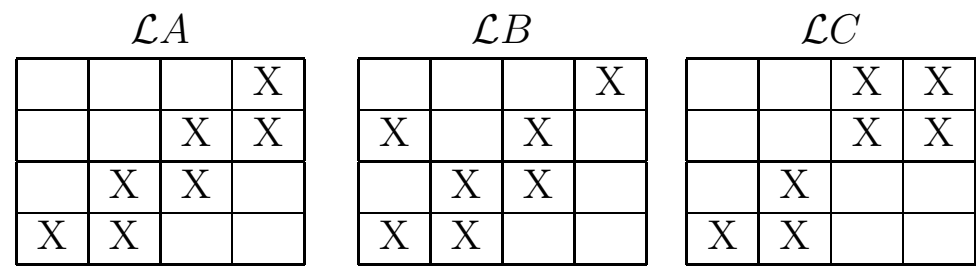

Figure 7: The three structures of $\mathcal{L}$ equivalent to an $(3,9,2){ }_{4} 1$ code. 


LA
\begin{tabular}{|c|c|c|c|}
\hline & 0 & & $\mathrm{X}$ \\
\hline & 1 & $\mathrm{X}$ & $\mathrm{X}$ \\
\hline 3 & $\mathrm{X}$ & $\mathrm{X}$ & 2 \\
\hline $\mathrm{X}$ & $\mathrm{X}$ & 2 & 3 \\
\hline
\end{tabular}

\begin{tabular}{l|c|c|c|}
\multicolumn{4}{c}{$\mathcal{L} B$} \\
\hline & 0 & & $\mathrm{X}$ \\
\hline $\mathrm{X}$ & 1 & $\mathrm{X}$ & \\
\hline 3 & $\mathrm{X}$ & $\mathrm{X}$ & 2 \\
\hline $\mathrm{X}$ & $\mathrm{X}$ & 2 & 3 \\
\hline
\end{tabular}

\begin{tabular}{|c|c|c|c|}
\multicolumn{5}{c}{$\mathcal{L} C$} \\
\hline 1 & 0 & $\mathrm{X}$ & $\mathrm{X}$ \\
\hline 0 & 1 & $\mathrm{X}$ & $\mathrm{X}$ \\
\hline $\mathrm{X}$ & $\mathrm{X}$ & 3 & 2 \\
\hline $\mathrm{X}$ & $\mathrm{X}$ & 2 & 3 \\
\hline
\end{tabular}

Figure 8: These partial Latin squares cannot be completed while retaining nonextendability.
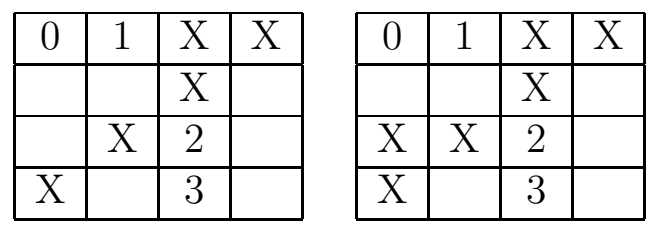

\begin{tabular}{|c|c|c|c|}
\hline 0 & 1 & $\mathrm{X}$ & $\mathrm{X}$ \\
\hline 1 & & $\mathrm{X}$ & \\
\hline $\mathrm{X}$ & $\mathrm{X}$ & 2 & 3 \\
\hline $\mathrm{X}$ & & 3 & \\
\hline
\end{tabular}

Figure 9: The features of $\mathcal{L}$ equivalent to a $(3,10,2)_{4} 1$ code.

For $\mathcal{L} C$ to ensure cells $(1,2)$ and $(1,3)$ cannot be filled cells $(2,2)$ and $(2,3)$ may be filled uniquely. From this row 2 and column 0 already contain all the symbols, thus cell $(0,2)$ must be empty.

Hence the non-extendable partial Latin squares $\mathcal{L} A, \mathcal{L} B$ and $\mathcal{L} C$ cannot be completed. There is no non-extendable partial Latin square with seven empty cells, hence there is no $(3,9,2)_{4} 1$ code.

Theorem 14. There are four non-equivalent $(3,10,2)_{4} 1$ codes.

Proof. Using Lemma 7 we know there must be two triangles and two isolated lines of each colour of the associated graph, $\mathcal{G}$. $\mathcal{L}$, the equivalent Latin square must have at least one empty cell in each row and column with two of the rows and columns having two empty cells. Without loss of generality we can choose row 0 , column 2 , which can then be completed. If we choose the other empty cell in row 1 or column 3 , then the nonextendable partial Latin square cannot be completed. Thus we must choose the other empty cell in row 2 or 3 , column 0 or 1 . Without loss of generality choose row 2 , column 0 , which can then be uniquely completed (figure 9 ).

There are now four cells left to fill in. There are four possible ways to do this (figure $10)$.

\begin{tabular}{|c|c|c|c|}
\multicolumn{4}{c}{$\mathcal{L} A$} \\
\hline 0 & 1 & $\mathrm{X}$ & $\mathrm{X}$ \\
\hline 1 & 0 & $\mathrm{X}$ & 2 \\
\hline $\mathrm{X}$ & $\mathrm{X}$ & 2 & 3 \\
\hline $\mathrm{X}$ & 2 & 3 & 0 \\
\hline
\end{tabular}

\begin{tabular}{|c|c|c|c|}
\multicolumn{4}{c}{$\mathcal{L} B$} \\
\hline 0 & 1 & $\mathrm{X}$ & $\mathrm{X}$ \\
\hline 1 & 0 & $\mathrm{X}$ & 2 \\
\hline $\mathrm{X}$ & $\mathrm{X}$ & 2 & 3 \\
\hline $\mathrm{X}$ & 2 & 3 & 1 \\
\hline
\end{tabular}

\begin{tabular}{|c|c|c|c|}
\multicolumn{4}{c}{$\mathcal{L} C$} \\
\hline 0 & 1 & $\mathrm{X}$ & $\mathrm{X}$ \\
\hline 1 & 2 & $\mathrm{X}$ & 0 \\
\hline $\mathrm{X}$ & $\mathrm{X}$ & 2 & 3 \\
\hline $\mathrm{X}$ & 0 & 3 & 2 \\
\hline
\end{tabular}

\begin{tabular}{|c|c|c|c|}
\multicolumn{4}{c}{$\mathcal{L} D$} \\
\hline 0 & 1 & $\mathrm{X}$ & $\mathrm{X}$ \\
\hline 1 & 3 & $\mathrm{X}$ & 0 \\
\hline $\mathrm{X}$ & $\mathrm{X}$ & 2 & 3 \\
\hline $\mathrm{X}$ & 0 & 3 & 2 \\
\hline
\end{tabular}

Figure 10: The four non-equivalent non-extendable partial Latin squares which are equivalent to $(3,10,2){ }_{4} 1$ codes. 


\begin{tabular}{|c|c|c|c|}
\hline 0 & & & $\mathrm{X}$ \\
\hline 1 & & $\mathrm{X}$ & \\
\hline $\mathrm{X}$ & $\mathrm{X}$ & 2 & 3 \\
\hline $\mathrm{X}$ & & & \\
\hline
\end{tabular}

Figure 11: The features of $\mathcal{L}$ equivalent to a $(3,11,2){ }_{4} 1$ code.

\begin{tabular}{|c|c|c|c|}
\hline 0 & 1 & $\mathrm{X}$ & $\mathrm{X}$ \\
\hline 1 & & $\mathrm{X}$ & \\
\hline $\mathrm{X}$ & $\mathrm{X}$ & 2 & 3 \\
\hline $\mathrm{X}$ & 0 & 3 & 2 \\
\hline
\end{tabular}

\begin{tabular}{|c|c|c|c|}
\hline 0 & & & $\mathrm{X}$ \\
\hline 1 & 0 & $\mathrm{X}$ & $\mathrm{X}$ \\
\hline $\mathrm{X}$ & $\mathrm{X}$ & 2 & 3 \\
\hline $\mathrm{X}$ & 1 & 3 & 2 \\
\hline
\end{tabular}

\begin{tabular}{|c|c|c|c|}
\hline 0 & 1 & $\mathrm{X}$ & $\mathrm{X}$ \\
\hline 1 & 0 & $\mathrm{X}$ & \\
\hline $\mathrm{X}$ & $\mathrm{X}$ & 2 & 3 \\
\hline $\mathrm{X}$ & 2 & 3 & \\
\hline
\end{tabular}

\begin{tabular}{|c|c|c|c|}
\hline 0 & 1 & & $\mathrm{X}$ \\
\hline 1 & 0 & $\mathrm{X}$ & $\mathrm{X}$ \\
\hline $\mathrm{X}$ & $\mathrm{X}$ & 2 & 3 \\
\hline $\mathrm{X}$ & 3 & & 2 \\
\hline
\end{tabular}

Figure 12: Each option of filling in cell $(3,1)$ requires another empty cell in $\mathcal{L}$

$\mathcal{L} A$ has two Latin subsquares, $\mathcal{L} B$ and $\mathcal{L} C$ have one Latin subsquare and $\mathcal{L} D$ has no Latin subsquares. Let $\mathcal{G} B$ and $\mathcal{G} C$ be the OQW graphs associated with $\mathcal{L} B$ and $\mathcal{L} C$. $\mathcal{G} C$ has four vertices of degree six, $112,130,332,310, \mathcal{G} B$ has only three vertices of degree six, 132, 331,312. $\mathcal{L} A, \mathcal{L} B, \mathcal{L} C$ and $\mathcal{L} D$ are non-equivalent. There are four non-equivalent $(3,10,2)_{4} 1$ codes.

Theorem 15. No $(3,11,2)_{4} 1$ code exists.

Proof. Using Lemma 7 we know there must be three triangles and one isolated line in each colour of $\mathcal{G}$. $\mathcal{L}$ the equivalent Latin square must have at least one empty cell in each row and column with one of the rows and columns having two empty cells. Without loss of generality we choose row 2 , column 0 , which can then be completed (figure 11).

We now try to fill in the remaining cells of the partial Latin square beginning with cell $(3,1)$. Each choice of entry for cell $(3,1)$ creates the need for another empty cell (figure 12).

There are no nonextendable partial Latin squares of side length three with five empty cells, thus there are no $(3,11,2){ }_{4} 1$ codes.

Example 16. There are many $(3,12,2){ }_{4} 1$ codes.

From Lemma 7, for each colour in the OQW-associated graph $\mathcal{G}$, there may be four triangles, or one 4-clique, two triangles and one isolated edge, or two 4-cliques and 2 isolated edges. This is too many structures for hand calculation. In figure 13 there are partial Latin squares equivalent to three $(3,12,2){ }_{4} 1$ codes, each of their OQW-associated graphs having a different number of triangles and 4-cliques.

Theorem 17. There are three non-equivalent $(3,13,2)_{4} 1$ codes.

Proof. Using Lemma 7 we know there may be three triangles and one 4-clique of each colour or two 4-cliques, one triangle and one isolated edge in $\mathcal{G}$. If there is one isolated edge then at least 4 empty cells are required (figure 14). In the equivalent partial Latin 


\begin{tabular}{|c|c|c|c|}
\hline 0 & 1 & 2 & $\mathrm{X}$ \\
\hline 1 & 0 & $\mathrm{X}$ & 3 \\
\hline 2 & $\mathrm{X}$ & 0 & 1 \\
\hline $\mathrm{X}$ & 3 & 1 & 0 \\
\hline
\end{tabular}

\begin{tabular}{|c|c|c|c|}
\hline 0 & 1 & 2 & $\mathrm{X}$ \\
\hline 1 & 3 & $\mathrm{X}$ & 0 \\
\hline 2 & $\mathrm{X}$ & 0 & 3 \\
\hline $\mathrm{X}$ & 0 & 3 & 2 \\
\hline
\end{tabular}

\begin{tabular}{|c|c|c|c|}
\hline 0 & 1 & 2 & $\mathrm{X}$ \\
\hline 1 & 2 & $\mathrm{X}$ & 3 \\
\hline 2 & $\mathrm{X}$ & 3 & 0 \\
\hline $\mathrm{X}$ & 3 & 0 & 1 \\
\hline
\end{tabular}

Figure 13: Three partial Latin squares which are equivalent to $(3,12,2){ }_{4} 1$ codes.

\begin{tabular}{|c|c|c|c|}
\hline & 0 & 1 & $\mathrm{X}$ \\
\hline 1 & & 0 & $\mathrm{X}$ \\
\hline & & & 2 \\
\hline & & & 3 \\
\hline
\end{tabular}

\begin{tabular}{|c|c|c|c|}
\hline 3 & 0 & 1 & $\mathrm{X}$ \\
\hline 1 & 2 & 0 & $\mathrm{X}$ \\
\hline 1 & 3 & $\mathrm{X}$ & 2 \\
\hline 2 & 0 & $\mathrm{X}$ & 3 \\
\hline
\end{tabular}

Figure 14: At least 4 empty cells are required.

square $\mathcal{L}$ we can fix column 0 , and have seven of the cells allocated. $\mathcal{L}$ has the structure of figure 15.

With the permutation $\mathrm{R}(12) \mathrm{C}(12) \mathrm{S}(12)$ squares with row 0 of $032 \mathrm{X}$ and $023 \mathrm{X}$ are equivalent to squares with row 0 of $013 \mathrm{X}$ and $031 \mathrm{X}$. Thus only squares with row 0 of 012X, 021X, 013X, or 031X are considered (figure 16).

- The following are equivalent to $\mathcal{L} A$ :

- $\mathcal{L} B$ using the permutation: (CS) $\mathrm{R}(01) \mathrm{C}(01)(23) \mathrm{S}(23)$.

- $\mathcal{L} D$ using the permutation: (CS) R(01) C(01)(23) S(123).

- $\mathcal{L} G$ using the permutation: (CS) $\mathrm{C}(23)$.

- $\mathcal{L} H$ using the permutation: $\mathrm{R}(01) \mathrm{C}(01) \mathrm{S}(01)$.

- $\mathcal{L} I$ using the permutation: (CSR) R(03)(12) C(0213) S(13).

- $\mathcal{L} L$ using the permutation: (RC) $\mathrm{R}(03)(12) \mathrm{C}(03)(12) \mathrm{S}(01)$.

- $\mathcal{L} M$ using the permutation: (RSC) R(0312) C(0312) S(2130).

- The following are equivalent to $\mathcal{L} J$ :

- $\mathcal{L} K$ using the permutation: (CS) R(021) C(03)(21) S(031).

- $\mathcal{L} O$ using the permutation: (RC) $\mathrm{R}(03)(12) \mathrm{C}(03)(12) \mathrm{S}(012)$.

- The following are equivalent to $\mathcal{L} E$ :

\begin{tabular}{|l|l|l|l|}
\hline 0 & & & $\mathrm{X}$ \\
\hline 1 & & $\mathrm{X}$ & \\
\hline 2 & $\mathrm{X}$ & & \\
\hline 3 & & & \\
\hline
\end{tabular}

Figure 15: The structure of $\mathcal{L}$ equivalent to a $(3,13,2){ }_{4} 1$ code. 


\begin{tabular}{|c|c|c|c|c|c|c|c|c|c|c|c|c|c|c|c|c|c|c|c|}
\hline \multicolumn{4}{|c|}{$\mathcal{L} A$} & \multicolumn{4}{|c|}{$\mathcal{L} B$} & \multicolumn{4}{|c|}{$\mathcal{L} C$} & \multicolumn{4}{|c|}{$\mathcal{L} D$} & \multicolumn{4}{|c|}{$\mathcal{L} E$} \\
\hline 0 & 1 & 2 & $\mathrm{X}$ & 0 & 1 & 2 & $\mathrm{X}$ & 0 & 1 & 2 & $\mathrm{X}$ & 0 & 1 & 2 & $\mathrm{X}$ & 0 & 2 & 1 & $\mathrm{X}$ \\
\hline 1 & 0 & $\mathrm{X}$ & 3 & 1 & 0 & $\mathrm{X}$ & 3 & 1 & 3 & $\mathrm{X}$ & 0 & 1 & 3 & $\mathrm{X}$ & 2 & 1 & 0 & $\mathrm{X}$ & 3 \\
\hline 2 & $\mathrm{X}$ & 3 & 0 & 2 & $\bar{X}$ & 3 & 1 & 2 & $\bar{X}$ & 0 & 3 & 2 & $\mathrm{X}$ & 0 & 3 & 2 & $\bar{X}$ & 3 & 1 \\
\hline 3 & 2 & 0 & 1 & 3 & 2 & 1 & 0 & 3 & 0 & 1 & 2 & 3 & 2 & 1 & 0 & 3 & 1 & 2 & 0 \\
\hline \multicolumn{4}{|c|}{$\mathcal{L} F$} & \multicolumn{4}{|c|}{$\mathcal{L} G$} & \multicolumn{4}{|c|}{$\mathcal{L} H$} & \multicolumn{4}{|c|}{$\mathcal{L} I$} & \multicolumn{4}{|c|}{$\mathcal{L} J$} \\
\hline 0 & 2 & 1 & $\mathrm{X}$ & 0 & 1 & 3 & $\mathrm{X}$ & 0 & 1 & 3 & $\mathrm{X}$ & 0 & 1 & 3 & $\mathrm{X}$ & 0 & 1 & 3 & $\mathrm{X}$ \\
\hline 1 & 3 & $\mathrm{X}$ & 2 & 1 & 0 & $\mathrm{X}$ & 2 & 1 & 0 & $\mathrm{X}$ & 2 & 1 & 2 & $\mathrm{X}$ & 0 & 1 & 3 & $\mathrm{X}$ & 2 \\
\hline 2 & $\mathrm{X}$ & 0 & 3 & 2 & $\mathrm{X}$ & 0 & 3 & 2 & $\bar{X}$ & 1 & 3 & 2 & $\mathrm{X}$ & 0 & 3 & 2 & $\mathrm{X}$ & 0 & 3 \\
\hline 3 & 1 & 2 & 0 & 3 & 2 & 1 & 0 & 3 & 2 & 0 & 1 & 3 & 0 & 1 & 2 & 3 & 0 & 2 & 1 \\
\hline \multicolumn{4}{|c|}{$\mathcal{L} K$} & \multicolumn{4}{|c|}{$\mathcal{L} L$} & \multicolumn{4}{|c|}{$\mathcal{L} M$} & \multicolumn{4}{|c|}{$\mathcal{L} N$} & \multicolumn{4}{|c|}{$\mathcal{L} O$} \\
\hline 0 & 1 & 3 & $\mathrm{X}$ & 0 & 1 & 3 & $\mathrm{X}$ & 0 & 1 & 3 & $\mathrm{X}$ & 0 & 3 & 1 & $\mathrm{X}$ & 0 & 3 & 1 & $\mathrm{X}$ \\
\hline 1 & 3 & $\mathrm{X}$ & 2 & 1 & 3 & $\mathrm{X}$ & $\overline{2}$ & 1 & 3 & $\mathrm{X}$ & 2 & 1 & 0 & $\mathrm{X}$ & 2 & 1 & 2 & $\mathrm{X}$ & 3 \\
\hline$\overline{2}$ & $\mathrm{X}$ & 0 & 3 & 2 & $\mathrm{X}$ & 1 & $\overline{0}$ & 2 & $\bar{X}$ & 0 & 1 & 2 & $\mathrm{X}$ & 3 & 1 & 2 & $\mathrm{X}$ & 3 & 0 \\
\hline 3 & 2 & 1 & 0 & 3 & 2 & 0 & 1 & 3 & 2 & 1 & 0 & 3 & 1 & 2 & 0 & 3 & 1 & 0 & 2 \\
\hline
\end{tabular}

Figure 16: The fifteen non-extendable partial Latin squares equivalent to $(3,13,2){ }_{4} 1$ codes.

\begin{tabular}{|l|l|l|l|}
\hline 0 & & & $\mathrm{X}$ \\
\hline 1 & & $\mathrm{X}$ & \\
\hline 2 & & & \\
\hline 3 & & & \\
\hline
\end{tabular}

Figure 17: The structure of $\mathcal{L}$ equivalent to a $(3,14,2)_{4} 1$ code.

- $\mathcal{L} C$ using the permutation: (RC) $\mathrm{R}(032) \mathrm{C}(013) \mathrm{S}(01)$.

- $\mathcal{L} F$ using the permutation: $\mathrm{R}(12) \mathrm{C}(12) \mathrm{S}(12)$.

- $\mathcal{L} N$ using the permutation: (RC) $\mathrm{R}(03) \mathrm{C}(03)$.

$\mathcal{L} A$ has one Latin subsquare. $\mathcal{L} E$ and $\mathcal{L} J$ have no Latin subsquares. The graph $\mathcal{G} J$ of the code equivalent to $\mathcal{L} J$ has one vertex, 303, of degree nine. The graph $\mathcal{G} E$ has no vertices of degree nine. $\mathcal{L} A, \mathcal{L} E$ and $\mathcal{L} J$ are non-equivalent; there are three non-equivalent $(3,13,2){ }_{4} 1$ codes.

Theorem 18. There are six non-equivalent $(3,14,2)_{4} 1$ codes.

Proof. Using Lemma 7 we know there must be two triangles and two 4-cliques of each colour in $\mathcal{G}$. We can fix the first column, in that way have six of the sixteen cells allocated. The equivalent Latin square $\mathcal{L}$ must have the structure of figure 17 .

Using the permutation $\mathrm{R}(23) \mathrm{S}(23)$ squares with row 0 of $012 \mathrm{X}, 021 \mathrm{X}$ and $023 \mathrm{X}$ are equivalent to squares with row 0 of $013 \mathrm{X}, 031 \mathrm{X}$ and $032 \mathrm{X}$ respectively. Thus only nonextendable partial Latin squares with row 0 of $012 \mathrm{X}, 021 \mathrm{X}$ and $023 \mathrm{X}$ are considered (figure 18). 


\begin{tabular}{|c|c|c|c|c|c|c|c|c|c|c|c|c|c|c|c|c|c|c|c|}
\hline \multicolumn{4}{|c|}{$\mathcal{L} A$} & \multicolumn{4}{|c|}{$\mathcal{L} B$} & \multicolumn{4}{|c|}{$\mathcal{L} C$} & \multicolumn{4}{|c|}{$\mathcal{L} D$} & \multicolumn{4}{|c|}{$\mathcal{L} E$} \\
\hline 0 & 1 & 2 & $\mathrm{X}$ & 0 & 1 & 2 & $\mathrm{X}$ & 0 & 1 & 2 & $\mathrm{X}$ & 0 & 1 & 2 & $\mathrm{X}$ & 0 & 1 & 2 & $\mathrm{X}$ \\
\hline 1 & 0 & $\mathrm{X}$ & 3 & 1 & 0 & $\mathrm{X}$ & 3 & 1 & 2 & $\mathrm{X}$ & 3 & 1 & 3 & $\mathrm{X}$ & 0 & 1 & 3 & $\mathrm{X}$ & 2 \\
\hline 2 & 3 & 1 & 0 & 2 & 3 & 0 & 1 & 2 & 3 & 0 & 1 & 2 & 0 & 1 & 3 & 2 & 0 & 1 & 3 \\
\hline 3 & 2 & 0 & 1 & 3 & 2 & 1 & $\overline{0}$ & 3 & 0 & 1 & 2 & 3 & 2 & 0 & 1 & 3 & 2 & 0 & 1 \\
\hline \multicolumn{4}{|c|}{$\mathcal{L} F$} & \multicolumn{4}{|c|}{$\mathcal{L} G$} & \multicolumn{4}{|c|}{$\mathcal{L} H$} & \multicolumn{4}{|c|}{$\mathcal{L} I$} & \multicolumn{4}{|c|}{$\mathcal{L} J$} \\
\hline 0 & 2 & 1 & $\mathrm{X}$ & 0 & 2 & 1 & $\mathrm{X}$ & 0 & 2 & 1 & $\mathrm{X}$ & 0 & 2 & 1 & $\mathrm{X}$ & 0 & 2 & 1 & $\mathrm{X}$ \\
\hline 1 & 0 & $\mathrm{X}$ & 3 & 1 & 3 & $\mathrm{X}$ & 0 & 1 & 3 & $\mathrm{X}$ & 0 & 1 & 3 & $\mathrm{X}$ & 2 & 1 & 3 & $\mathrm{X}$ & 2 \\
\hline 2 & 3 & 0 & 1 & 2 & 1 & 0 & 3 & 2 & 0 & 3 & 1 & 2 & 0 & 3 & 1 & 2 & 1 & 0 & 3 \\
\hline 3 & 1 & 2 & 0 & 3 & 0 & 2 & 1 & 3 & 1 & 0 & 2 & 3 & 1 & 2 & 0 & 3 & 0 & 2 & 1 \\
\hline \multicolumn{4}{|c|}{$\mathcal{L} K$} & \multicolumn{4}{|c|}{$\mathcal{L} L$} & \multicolumn{4}{|c|}{$\mathcal{L} M$} & \multicolumn{4}{|c|}{$\mathcal{L} N$} & \multicolumn{4}{|c|}{$\mathcal{L} O$} \\
\hline 0 & 2 & 3 & $\mathrm{X}$ & 0 & 2 & 3 & $\mathrm{X}$ & 0 & 2 & 3 & $\mathrm{X}$ & 0 & 2 & 3 & $\mathrm{X}$ & 0 & 2 & 3 & $\mathrm{X}$ \\
\hline 1 & 0 & $\mathrm{X}$ & 2 & 1 & 0 & $\mathrm{X}$ & 3 & 1 & 0 & $\mathrm{X}$ & 3 & 1 & 3 & $\mathrm{X}$ & 0 & 1 & 3 & $\mathrm{X}$ & 2 \\
\hline 2 & 3 & 0 & 1 & 2 & 3 & 0 & 1 & 2 & 3 & 1 & 0 & 2 & 1 & 0 & 3 & 2 & 1 & 0 & 3 \\
\hline 3 & 1 & 2 & 0 & 3 & 1 & 2 & 0 & 3 & 1 & 0 & 2 & 3 & 0 & 2 & 1 & 3 & 0 & 2 & 1 \\
\hline
\end{tabular}

Figure 18: The fifteen different non-extendable partial Latin squares which are equivalent to $(3,14,2)_{4} 1$ codes.

- $\mathcal{L} A \equiv \mathcal{L} B$ using the permutation: $\mathrm{R}(01)(23) \mathrm{C}(32) \mathrm{S}(01)(32)$.

- $\mathcal{L} F \equiv \mathcal{L} C$ using the permutation: (RC) R(1203) C(0213) S(012).

- $\mathcal{L} K \equiv \mathcal{L} D$ using the permutation: $\mathrm{C}(01) \mathrm{S}(0132)$.

- The following are equivalent to $\mathcal{L} E$ :

- $\mathcal{L} G$ using the permutation: (CS) C(01) S(012).

- $\mathcal{L} J$ using the permutation: (RS) R(13) S(13).

- $\mathcal{L} L$ using the permutation: $\mathrm{C}(01) \mathrm{S}(0132)$.

- $\mathcal{L} N$ using the permutation: (RS) R(12) C(12) S(321).

- $\mathcal{L} O$ using the permutation: (CS) R(23) C(03)(12) S(02).

- $\mathcal{L} M \equiv \mathcal{L} H$ using the permutation: (CS) $\mathrm{C}(13) \mathrm{R}(23)$.

$\mathcal{L} A$ has three Latin subsquares, $\mathcal{L} D, \mathcal{L} H$ and $\mathcal{L} I$ have one Latin subsquare, $\mathcal{L} E$ and $\mathcal{L} C$ have no Latin subsquares. $\mathcal{G} C$ the graph OQW-associated with $\mathcal{L} C$ has one vertex of degree nine, 202; $\mathcal{G} E$ has two vertices of degree nine, 202,312. $\mathcal{G} H$ has two adjacent vertices of degree nine, 211,310; $\mathcal{G} I$ has two non-adjacent vertices of degree nine, 202, 311; $\mathcal{G} D$ has one vertex of degree nine, 210. Thus $\mathcal{L} B, \mathcal{L} C, \mathcal{L} E, \mathcal{L} D, \mathcal{L} H$ and $\mathcal{L} I$ are nonequivalent; there are six non-equivalent $(3,14,2) 4_{1}$ codes.

Theorem 19. No $(3,15,2)_{4} 1$ code exists. 
Proof. Assume $\mathcal{C}$ is a $(3,15,2)_{4} 1$ code. From Lemma 7 we know that $\mathcal{G}$, the OQWassociated graph, has three 4-cliques and one triangle of each colour, 63 edges in all. Using equation (1) we require 40 multicoloured triangles in $\mathcal{G}$.

If every edge of an $\alpha$-coloured 4 -clique is in two multicoloured triangles then $\mathcal{G}$ has four 4-cliques of colours $\beta$ and $\gamma$. This requires sixteen vertices, so every single coloured 4-clique must have at least one edge in less than two multicoloured triangles. The number of multicoloured triangles in $\mathcal{G}$,

$T \leq \frac{2}{3}(63-9)+\frac{1}{3} 9=39$. $\mathcal{C}$ is not a $(3,15,2){ }_{4} 1$ code.

Theorem 20. There are two non-equivalent $(3,16,2){ }_{4} 1$ codes.

Proof. There are two non-equivalent $(16,9,4,6)$ strongly regular graphs [11]. Both may be coloured such that every edge is in two multicoloured triangles. There are two nonequivalent Latin squares of side length 4 [3].

\section{Acknowledgements}

The author would like to thank Brendan McKay for several useful conversations and the anonymous referee for valuable suggestions.

\section{References}

[1] A. Blokhuis, S. Enger, H.D.L. Hollmann, and J.H. van Lint. On codes with covering radius 1 and minimum distance 2. Indagationes Mathematical, 12(4):449-452, 2001.

[2] G. Cohen, I. Honkala, S. Litsyn, and A. Lobstein. Covering Codes. North Holland, 1997.

[3] C. J. Colbourn, J. H. Dinitz, and I. M. Wanless. Handbook of combinatorial designs, chapter Latin Squares, pages 135-151. Chapman \& Hall/CRC, 2nd edition, 2007.

[4] P. Delsarte. Four fundamental parameters of a code and their combinatorial significance. Information and Control, 23:407-438, 1973.

[5] W. Haas and J. Quistorff. On mixed codes with covering radius 1 and minimum distance 2. The Electronic Journal of Combinatorics, 14:R51, 2007.

[6] J. L. Hall. Graphical associations and codes with small covering radius. Master's thesis, Australian National University, July 2007.

[7] R. W. Hamming. Error detecting and error correcting codes. Bell System Tech. J., 29:147-160, 1950.

[8] J. W. P. Hirschfeld and L. Storme. The packing problem in statistics coding theory and finite projective spaces. Journal of Statistical Planning and Inference, 72:355380, 1998. 
[9] P. R. J. Östergård, J. Quistorff, and A. Wassermann. New results on codes with covering radius 1 and minimum distance 2. Designs, Codes and Cryptography, 35:241$250,2005$.

[10] J. Quistorff. On codes with a given minimum distance and covering radius. Beiträge zur Algebra und Geometrie, 42(2):601-611, 2001.

[11] T. Spence. Strongly regular graphs on at most 64 vertices. www.maths.gla.ac.uk/ es/srgraphs.html, 1/12/2007.

[12] R.G. Stanton, J.D. Horton, and J.G. Kalbfleisch. Covering theorems for vectors with special reference to the case of four and five components. Journal of the London Mathematical Society, pages 493-499, 1969. 


\section{Corrigendum - submitted 4 Jun 2008}

Theorem 14 in its original form is incorrect. The correct proof is given below.

Theorem 14. Revised There are three non-equivalent $(3,10,2)_{4} 1$ codes.

Proof. Using Lemma 7 we know there must be two triangles and two isolated lines of each colour or three triangles and an isolated vertex in each single coloured subgraph of the OQW-associated graph, $\mathcal{G}$.

If $\mathcal{G}$ has two triangles of each colour then $\mathcal{L}$, the equivalent Latin square must have at least one empty cell in each row and column, with two of the rows and columns having two empty cells. Without loss of generality we can choose row 0 , column 2 , to have 2 empty cells; the remaining cells in row 0 and column 2 can be uniquely filled (up to equivalence). If we choose the other empty cell in row 1 or column 3 , then the non-extendable partial Latin square cannot be completed. Thus we must choose the other empty cell in row 2 or 3 , column 0 or 1 . Without loss of generality choose row 2, column 0 to have another empty cell; the remaining cells in row 2 and column 3 can be uniquely filled (figure 19).

\begin{tabular}{|c|c|c|c|}
\hline 0 & 1 & $\mathrm{X}$ & $\mathrm{X}$ \\
\hline & & $\mathrm{X}$ & \\
\hline & $\mathrm{X}$ & 2 & \\
\hline $\mathrm{X}$ & & 3 & \\
\hline
\end{tabular}

\begin{tabular}{|c|c|c|c|}
\hline 0 & 1 & $\mathrm{X}$ & $\mathrm{X}$ \\
\hline & & $\mathrm{X}$ & \\
\hline $\mathrm{X}$ & $\mathrm{X}$ & 2 & \\
\hline $\mathrm{X}$ & & 3 & \\
\hline
\end{tabular}

\begin{tabular}{|c|c|c|c|}
\hline 0 & 1 & $\mathrm{X}$ & $\mathrm{X}$ \\
\hline 1 & & $\mathrm{X}$ & \\
\hline $\mathrm{X}$ & $\mathrm{X}$ & 2 & 3 \\
\hline $\mathrm{X}$ & & 3 & \\
\hline
\end{tabular}

Figure 19: The features of $\mathcal{L}$ equivalent to a $(3,10,2)_{4} 1$ code with two triangles of each colour in its OQW-associated graph.

There are now four cells left to fill in. There are four possible ways to do this (figure 20).

\begin{tabular}{|c|c|c|c|}
\multicolumn{4}{c}{$\mathcal{L} A$} \\
\hline 0 & 1 & $\mathrm{X}$ & $\mathrm{X}$ \\
\hline 1 & 0 & $\mathrm{X}$ & 2 \\
\hline $\mathrm{X}$ & $\mathrm{X}$ & 2 & 3 \\
\hline $\mathrm{X}$ & 2 & 3 & 0 \\
\hline
\end{tabular}

\begin{tabular}{|c|c|c|c|}
\multicolumn{4}{c}{$\mathcal{L} B$} \\
\hline 0 & 1 & $\mathrm{X}$ & $\mathrm{X}$ \\
\hline 1 & 0 & $\mathrm{X}$ & 2 \\
\hline $\mathrm{X}$ & $\mathrm{X}$ & 2 & 3 \\
\hline $\mathrm{X}$ & 2 & 3 & 1 \\
\hline
\end{tabular}

\begin{tabular}{|c|c|c|c|}
\multicolumn{4}{c}{$\mathcal{L} C$} \\
\hline 0 & 1 & $\mathrm{X}$ & $\mathrm{X}$ \\
\hline 1 & 2 & $\mathrm{X}$ & 0 \\
\hline $\mathrm{X}$ & $\mathrm{X}$ & 2 & 3 \\
\hline $\mathrm{X}$ & 0 & 3 & 2 \\
\hline
\end{tabular}

\begin{tabular}{|c|c|c|c|}
\multicolumn{4}{c}{$\mathcal{L} D$} \\
\hline 0 & 1 & $\mathrm{X}$ & $\mathrm{X}$ \\
\hline 1 & 3 & $\mathrm{X}$ & 0 \\
\hline $\mathrm{X}$ & $\mathrm{X}$ & 2 & 3 \\
\hline $\mathrm{X}$ & 0 & 3 & 2 \\
\hline
\end{tabular}

Figure 20: Four non-extendable partial Latin squares which are equivalent to $(3,10,2){ }_{4} 1$ codes with two triangles of each colour in their OQW-associated graph.

$\mathcal{L} A$ and $\mathcal{L} C$ have two Latin subsquares, $\mathcal{L} B$ and $\mathcal{L} D$ have one Latin subsquare. $\mathcal{L} A$ is equivalent to $\mathcal{L} C$ and $\mathcal{L} B$ is equivalent to $\mathcal{L} D$, both using the permutation: $\mathrm{R}(02)(13)$ $\mathrm{C}(02)(13) \mathrm{S}(02)(13)$.

If $\mathcal{G}$ has 3 triangles and one isolated vertex of colour $\alpha$ then there must be three empty cells in the same row, we choose row 0 as in figure 21. If there are any other empty cells in columns 1,2 or 3 , then a cell in row 0 marked as being empty may be filled. Thus the 3 other empty cells must be in column 0 . Choose a symbol for cell $(0,0)$, then the 
rest of the square may be filled by a Latin square of side length 3 as in $\mathcal{L} E$. There is only one reduced Latin square of side length 3 [3]. Any other Latin square is obtained by permuting rows 1,2 and 3 and columns 1, 2 and 3 giving an equivalent non-extendable partial Latin square.

\begin{tabular}{|l|l|l|l|}
\hline & $\mathrm{X}$ & $\mathrm{X}$ & $\mathrm{X}$ \\
\hline & & & \\
\hline & & & \\
\hline & & & \\
\hline
\end{tabular}

\begin{tabular}{|l|l|l|l|}
\hline & $\mathrm{X}$ & $\mathrm{X}$ & $\mathrm{X}$ \\
\hline $\mathrm{X}$ & & & \\
\hline $\mathrm{X}$ & & & \\
\hline $\mathrm{X}$ & & & \\
\hline
\end{tabular}

\begin{tabular}{|c|c|c|c|}
\multicolumn{4}{c}{$\mathcal{L} E$} \\
\hline 0 & $\mathrm{X}$ & $\mathrm{X}$ & $\mathrm{X}$ \\
\hline $\mathrm{X}$ & 1 & 2 & 3 \\
\hline $\mathrm{X}$ & 2 & 3 & 1 \\
\hline $\mathrm{X}$ & 3 & 1 & 2 \\
\hline
\end{tabular}

Figure 21: A partial Latin square equivalent to a $(3,10,2){ }_{4} 1$ code that has three triangles of each colour in its OQW-associated graph

There are three non-equivalent $(3,10,2){ }_{4} 1$ codes. 